\title{
Magnetic Exchange Couplings from Noncollinear Spin Density Functional Perturbation Theory
}

\author{
Juan E. Peralta and Veronica Barone \\ Department of Physics, Central Michigan University, Mt. Pleasant, MI 48859
}

(Dated: November 10, 2018)

\begin{abstract}
We propose a method for the evaluation of magnetic exchange couplings based on noncollinear spin-density functional calculations. The method employs the second derivative of the total KohnSham energy of a single reference state, in contrast to approximations based on Kohn-Sham total energy differences. The advantage of our approach is twofold: It provides a physically motivated picture of the transition from a low-spin to a high-spin state, and it utilizes a perturbation scheme for the evaluation of magnetic exchange couplings. The latter simplifies the way these parameters are predicted using first-principles: It avoids the non-trivial search for different spin-states that needs to be carried out in energy difference methods and it opens the possibility of "black-boxifying" the extraction of exchange couplings from density functional theory calculations. We present proof of concept calculations of magnetic exchange couplings in the $\mathrm{H}-\mathrm{He}-\mathrm{H}$ model system and in an oxovanadium bimetallic complex where the results can be intuitively rationalized.
\end{abstract}

\section{INTRODUCTION}

Empirical models based on the Heisenberg spin Hamiltonian are routinely utilized to describe the behavior of a variety of magnetic systems. In most cases, these simple models are found to fit the experimental data very well, provided that the parameters in the model Hamiltonian are chosen properly. The set of parameters can include both, external parameters (temperature, applied magnetic field, etc.), and internal parameters (magnetic exchange couplings, magnetic anisotropy, etc.). Internal parameters for a particular system can be obtained either by fitting experimental data or from firstprinciples electronic structure calculations by mapping total electronic energies to the energies of the Heisenberg spin Hamiltonian, $\stackrel{1,2,3}{=}$ In particular, magnetic exchange couplings, $J$, can be obtained considering the isotropic Heisenberg Hamiltonian

$$
\hat{H}=-2 \sum_{<i, j>} J_{i j} \hat{\mathbf{S}}_{i} \cdot \hat{\mathbf{S}}_{j}
$$

where $\hat{\mathbf{S}}_{i}$ and $\hat{\mathbf{S}}_{j}$ are the (localized) spin operators associated to each magnetic center.

Perhaps the one of the most interesting manifestation of magnetism at the molecular scale can be found in complexes containing transition metal atoms. Many applications have been suggested exploiting these molecular-size magnets, such as quantum computation units and highdensity data storage. $\stackrel{\underline{4}}{=}$ Due to the relatively large size of most complexes of interest, density functional theory $(\mathrm{DFT})^{5}, \underline{6}$ offers the most efficient alternative for modeling the electronic structure of these systems from firstprinciples $, 3,7,8$

Several approaches had been proposed to extract $J$ couplings from DFT energies. According to the spinprojected (SP) approach,,$\frac{1,9,10}{1}$ the energies of a twocenter complex $A$ and $B$ can be related to the $J$ coupling as

$$
E_{L S}-E_{H S}=4 S_{A} S_{B} J_{A B},
$$

while in the non-projected (NP) approach ${ }^{11}$, the energies of a two-center complex $S_{A}$ and $S_{B}$ can be related to the $J$ coupling as

$$
E_{L S}-E_{H S}=\left(4 S_{A} S_{B}+2 S_{B}\right) J_{A B}
$$

where $S_{B} \leq S_{A}$. In Eqs. (21) and (3), $E_{H S}$ is the energy of the high-spin state and $E_{L S}$ is the energy of the low-spin (broken-symmetry) state. Eqs. (2) and (3) can be straightforwardly generalized to a set of equations for complexes with multiple magnetic centers $\stackrel{12,13}{\underline{1}}$ While the SP and NP methods are fairly popular, other methods have been proposed in the literature such as Nishino's approach 14 , the constrained-DFT approach of Rudra et al. 15,16, the Slater's transition state method of Dai and Whangbo 17 and the local spin method of Clark and Davidson ${ }^{18,19}$ All these approaches rely on the evaluation of the energy difference between two (for the simplest case of a bimetallic complex) or more states. The evaluation of this energy difference is commonly done by carrying out several self-consistent field calculations, one for each different magnetic configuration. However, in many cases, converging to the right target state could be cumbersome, specially for systems containing multiple centers with many magnetic configurations. Therefore, developing an approach that can be used in a "blackbox" manner is crucial to systematically explore a large set of complexes or complexes containing many magnetic centers.

In this work, we present an approach for the evaluation of magnetic exchange couplings based on noncollinear spin density functional calculations that allows, in analogy to response properties, to express the magnetic exchange couplings as a derivative of the total electronic energy of one single state with respect to an external parameter, opening the possibility of "black-boxifying" the extraction of magnetic exchange couplings from density functional theory calculations. 


\section{THEORY AND IMPLEMENTATION}

\section{A. Exchange Couplings as Energy Derivatives}

Let us consider the effective interaction energy between two magnetic centers $A$ and $B$ given by the isotropic classical Heisenberg model (obtained by taking the expectation value of Eq. (1)),

$$
\begin{aligned}
E_{A B} & =-2 J_{A B} \mathbf{S}_{A} \cdot \mathbf{S}_{B} \\
& =-2 J_{A B} S_{A} S_{B} \cos \theta,
\end{aligned}
$$

where $\mathbf{S}_{A}$ and $\mathbf{S}_{B}$ are the (perfectly localized) magnetic moment vectors, $J_{A B}$ is the exchange coupling constant, and $\theta$ is the angle between $\mathbf{S}_{A}$ and $\mathbf{S}_{B}$. From Eq. (4), one can trivially obtain $J_{A B}$ from the second derivative of $E_{A B}$ with respect to $\theta$ at the equilibrium points,

$$
\begin{aligned}
J_{A B} & =\frac{1}{2 S_{A} S_{B}}\left(\frac{d^{2} E_{A B}}{d \theta^{2}}\right)_{\theta=0} \\
& =-\frac{1}{2 S_{A} S_{B}}\left(\frac{d^{2} E_{A B}}{d \theta^{2}}\right)_{\theta=180^{\circ}} .
\end{aligned}
$$

These simple relations provide a direct path to the evaluation of magnetic exchange couplings $J_{A B}$ from density functional calculations if $E_{A B}$ in Eq. (15) is replaced by the total Kohn-Sham $(\mathrm{KS})^{20}$ energy of the system, $E^{K S}$. Therefore, assuming that the electronic system depends on $\theta$ as an ideal Heisenberg model (the validity of this assumption will be discussed in the next Section), one can express the exchange coupling constant $J_{A B}$ in terms of an energy derivative as

$$
J_{A B}=\frac{1}{2 S_{A} S_{B}}\left(\frac{d^{2} E^{K S}}{d \theta^{2}}\right)_{\theta=0}
$$

or

$$
J_{A B}=-\frac{1}{2 S_{A} S_{B}}\left(\frac{d^{2} E^{K S}}{d \theta^{2}}\right)_{\theta=180^{\circ}},
$$

where the angle $\theta$ in the DFT framework is defined as the angle between the local magnetization vectors $\mathbf{S}_{A}$ and $\mathbf{S}_{B}$. Another related method based on the Green's function formalism for crystals has been proposed by Liechtenstein et al. 21

\section{B. Constraint Noncollinear Spin-DFT Calculations}

To evaluate the dependence of $E^{K S}$ on $\theta$, we first introduce two-component spinors as Kohn-Sham orbitals,

$$
\Psi_{i}(\mathbf{r})=\left(\begin{array}{c}
\psi_{i}^{\uparrow}(\mathbf{r}) \\
\psi_{i}^{\downarrow}(\mathbf{r})
\end{array}\right)
$$

where $\psi_{i}^{\uparrow}(\mathbf{r})$ and $\psi_{i}^{\downarrow}(\mathbf{r})$ are spatial orbitals expanded in a linear combination of atomic orbitals,

$$
\psi_{i}^{\omega}(\mathbf{r})=\sum_{\mu} c_{\mu i}^{\omega} \phi_{\mu}(\mathbf{r})(\omega=\uparrow, \downarrow) .
$$

The two-component spinors introduce the freedom in the spin-dependence of the KS system that allows for local rotations of the spin density characterized by $\theta \neq 0$ and $\theta \neq 180^{\circ}$, i.e. noncollinear spin densities $, 22,23,24,25,26$ The local magnetization vectors $\mathbf{S}_{A}$ and $\mathbf{S}_{B}$ can be written as

$$
\mathbf{S}_{A, B}=\int d^{3} r \mathcal{W}_{A, B}(\mathbf{r}) \mathbf{s}(\mathbf{r}),
$$

where

$$
\mathbf{s}(\mathbf{r})=\sum_{i \in o c c} \Psi_{i}^{\dagger}(\mathbf{r}) \boldsymbol{\sigma} \Psi_{i}(\mathbf{r})
$$

is the spin-density vector and $\mathcal{W}_{A, B}(\mathbf{r})$ is a scalar weight function that determines each local magnetic site. It is important to recall that the magnetic centers $A$ and $B$ represent a group of one or more atoms. In Eq. (11), $\boldsymbol{\sigma}=\left(\sigma_{x}, \sigma_{y}, \sigma_{z}\right)$ is the $2 \times 2$ Pauli matrices vector.

Having defined the local magnetic moments, the second step is to find the dependence of the total electronic energy upon local rotations of the spin density. This is done by constraining the direction of the local magnetizations $\mathbf{S}_{A}$ and $\mathbf{S}_{B}$ by means of the Lagrange multipliers technique. To this end, we construct the Lagrangian functional $\Lambda$

$$
\begin{array}{r}
\Lambda\left[\{\Psi(\mathbf{r})\}, \boldsymbol{\lambda}_{A}, \boldsymbol{\lambda}_{B}\right]=E^{K S}[\{\Psi(\mathbf{r})\}]- \\
\boldsymbol{\lambda}_{A} \cdot\left(\mathbf{S}_{A} \times \hat{\mathbf{z}}\right)-\boldsymbol{\lambda}_{B} \cdot\left(\mathbf{S}_{B} \times \hat{\mathbf{e}}_{\theta}\right),
\end{array}
$$

where $\hat{\mathbf{e}}_{\theta}=\sin \theta \hat{\mathbf{x}}+\cos \theta \hat{\mathbf{z}}$ is a unity vector to which $\mathbf{S}_{B}$ is constraint to be parallel to, and for simplicity $\mathbf{S}_{A}$ has been chosen to be constraint to the $z$ direction, as schematized in Fig. 1. Here $0 \leq \theta \leq 180^{\circ}$ is considered as an external parameter for which $(d E / d \theta)_{\theta=0}=$ $(d E / d \theta)_{\theta=180^{\circ}}=0$. Eq. (12) can be readily generalized for the case of many magnetic centers and arbitrary unity vector directions. For the case of two magnetic centers (and for the purpose of this work), Eq. (12) does not imply any loss of generality. In Eq. (12), $E^{K S}[\{\Psi(\mathbf{r})\}]$ represents the KS energy of the system which is, in practice, a functional of the set of occupied KS orbitals $\{\Psi(\mathbf{r})\}$. Stationary solutions of $\Lambda$ for a given (fixed) $\theta$ imply

$$
\begin{gathered}
\frac{d \Lambda}{d \boldsymbol{\lambda}_{A}}=\mathbf{S}_{A} \times \hat{\mathbf{z}}=\mathbf{0}, \\
\frac{d \Lambda}{d \boldsymbol{\lambda}_{B}}=\mathbf{S}_{B} \times \hat{\mathbf{e}}_{\theta}=\mathbf{0},
\end{gathered}
$$

and

$$
\begin{gathered}
\frac{\delta \Lambda}{\delta \Psi_{i}^{\dagger}(\mathbf{r})}=\frac{\delta E^{K S}}{\delta \Psi_{i}^{\dagger}(\mathbf{r})}-\left[\mathcal{W}_{A}(\mathbf{r}) \boldsymbol{\lambda}_{A} \cdot(\boldsymbol{\sigma} \times \hat{\mathbf{z}})+\right. \\
\left.\mathcal{W}_{B}(\mathbf{r}) \boldsymbol{\lambda}_{B} \cdot\left(\boldsymbol{\sigma} \times \hat{\mathbf{e}}_{\theta}\right)\right] \Psi_{i}(\mathbf{r})=0(i \in \text { occ })
\end{gathered}
$$

While Eqs. (13) and (14) restore the constraint conditions, Eq. (15) combined with the orthonormality condition for the spinors yields a modified set of KS equations 
(in terms of two-component spinors) that include the two additional terms inside the square brackets on the lefthand side of Eq. (15),

$$
\begin{array}{r}
{\left[T+V_{N}+J+V_{x c}-\mathcal{W}_{A}(\mathbf{r}) \boldsymbol{\lambda}_{A} \cdot(\boldsymbol{\sigma} \times \hat{\mathbf{z}})-\right.} \\
\left.\mathcal{W}_{B}(\mathbf{r}) \boldsymbol{\lambda}_{B} \cdot\left(\boldsymbol{\sigma} \times \hat{\mathbf{e}}_{\theta}\right)\right] \Psi_{i}(\mathbf{r})=\epsilon_{i} \Psi_{i}(\mathbf{r}),
\end{array}
$$

where $T=-1 / 2 \nabla^{2}$ is the kinetic energy, $V_{N}$ is the electron-nuclei potential, $J$ is the Coulomb (or Hartree) potential, and $V_{x c}$ is the exchange-correlation (XC) potential. The sum of the first four terms inside the square brackets is the standard KS Hamiltonian, while the two additional terms can be interpreted as a potential originated in a torque exerted on the local magnetic moments $\mathbf{S}_{A}$ and $\mathbf{S}_{B}$. It should be noted that other approaches had been proposed in the literature to constraint the direction of the local mangetization in spin DFT calculations,$\underline{27,28,29}$

It is important to note that since the constraint conditions are linear in the spin density vectors, the additional terms in Eq. (16) depend implicitly on the orbitals only through $\boldsymbol{\lambda}_{A}$ and $\boldsymbol{\lambda}_{B}$, which simplifies the implementation.

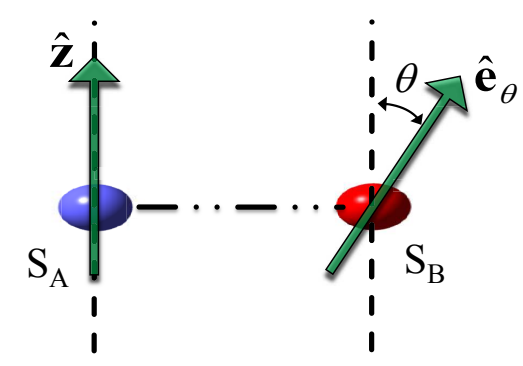

FIG. 1: Schematic representation of the constraint vectors employed for the local rotations of the spin density.

Neglecting spin-orbit interaction, $T, V_{N}$, and $J$ are diagonal in the $2 \times 2$ spin space, and thus the only term in Eq. (16) that couples $\psi_{i}^{\uparrow}$ and $\psi_{i}^{\downarrow}$ is $V_{x c}$. In a previous work, we have generalized $V_{x c}$ for noncollinear magnetizations,,$\underline{30}$ assuming that the XC energy depends on the local variables in the same manner as in the standard collinear (spin-unrestricted) case, and imposing the condition for the XC energy to be invariant under rigid rotations of the spin density. In that work, we have derived $V_{x c}$ for general energy functionals containing a variety of ingredients beyond the local-spin density approximation (LSDA) and the generalized-gradient approximation (GGA), such as meta-GGAs and hybrid density functionals. This same generalization is adopted throughout this work. Other implementations based on plane-waves ${ }^{22,23,24,25}$ and Gaussian-type orbitals can be found in the literature for a variety of applications $26,31,32,33,34,35$

The constraint vectors can be chosen without loss of generality to lay in the $x-z$ plane. Hence, as spin-orbit interaction is not included in the Hamiltonian, the twocomponent spinors are purely real. As a consequence, in this scheme the orbital magnetization of the solutions is always zero.

The value of $\Lambda$ at the stationary solutions for a fixed $\theta$ given by Eqs. (13) - (15) can be directly associated to the energy of the KS system in the presence of the constraints, $E^{K S}(\theta)$. The only arbitrariness in the formulation is the choice of the weight function $\mathcal{W}_{A, B}(\mathbf{r})$ and the fragments $A$ and $B$. Since in practice it is necessary to evaluate the matrix elements of $\mathcal{W}_{A, B}(\mathbf{r})$ in the atomic orbitals basis set $\phi_{\xi}(\mathbf{r})$, it is convenient to define $\mathbf{S}_{A}$ and $\mathbf{S}_{B}$ using population analysis,

$$
\mathbf{S}_{A, B}=\sum_{\mu, \nu}\left(\mathcal{W}_{A, B}\right)_{\mu \nu} \mathbf{P}_{\mu \nu}
$$

where $\mathbf{P}_{\mu \nu}$ is the spin-density matrix vector whose Cartesian components are

$$
\begin{aligned}
& P_{\mu \nu}^{x}=P_{\mu \nu}^{\uparrow \downarrow}+P_{\mu \nu}^{\downarrow \uparrow}, \\
& P_{\mu \nu}^{y}=i\left(P_{\mu \nu}^{\uparrow \downarrow}-P_{\mu \nu}^{\downarrow \uparrow}\right),
\end{aligned}
$$

and

$$
P_{\mu \nu}^{z}=P_{\mu \nu}^{\uparrow \uparrow}+P_{\mu \nu}^{\downarrow \downarrow}
$$

written in terms of the $2 \times 2$ density matrix

$$
P_{\mu \nu}^{\omega \omega^{\prime}}=\sum_{i \in \mathrm{OCC}} c_{\mu i}^{\omega} c_{\nu i}^{\omega^{\prime} *} \quad\left(\omega, \omega^{\prime}=\uparrow, \downarrow\right)
$$

For this work, we employ Löwdin population analysis $\underline{36}$ From the expression for the atomic spin-population given by Eq. (17), $\left(\mathcal{W}_{A, B}\right)_{\mu \nu}$ can be obtained as

$$
\left(\mathcal{W}_{A, B}\right)_{\mu \nu}=\frac{d S_{A, B}^{\eta}}{d P_{\mu \nu}^{\eta}} \quad(\eta=x, y \text { or } z) .
$$

Thus, using the Löwdin partitioning, it is straightforward to show that the matrix elements of $\mathcal{W}_{A, B}(\mathbf{r})$ can be calculated from the atomic orbitals overlap matrix $(\mathbb{S})_{\lambda \sigma}=\int d^{3} r \phi_{\lambda}(\mathbf{r}) \phi_{\sigma}(\mathbf{r})$ as

$$
\left(\mathcal{W}_{A, B}\right)_{\mu \nu}=\sum_{\lambda \in A, B}\left(\mathbb{S}^{1 / 2}\right)_{\lambda \mu}\left(\mathbb{S}^{1 / 2}\right)_{\nu \lambda} .
$$

It is worth commenting that atomic spin-densities are less sensitive to the choice of the population method than atomic densities $\frac{37}{}$ Since only the direction of the atomic spin-density is relevant in our method for the calculation of magnetic exchange couplings, we expect even less sensitivity with the coice of the population method.

The set of spinors that simultaneously satisfy Eq. (16) and Eqs. (13) and (14) needs to be determined selfconsistently since $J$ and $V_{x c}$ depend on the spinors. To obtain the KS Hamiltonian, we add the additional constraint terms of Eq. (16) to the standard KS Hamiltonian, initially using a guess for the Lagrange multipliers 
$\boldsymbol{\lambda}_{A}$ and $\boldsymbol{\lambda}_{B}$. Then we determine the optimal $\boldsymbol{\lambda}_{A}$ and $\boldsymbol{\lambda}_{B}$ such that Eqs. (13) and (14) are satisfied, using the density matrix obtained from diagonalizing the KS Hamiltonian of the constraint system to evaluate $\mathbf{S}_{A}$ and $\mathbf{S}_{B}$. This is carried out using the steepest descent method to find the minimum of $\Lambda$ as a function of $\boldsymbol{\lambda}_{A}$ and $\boldsymbol{\lambda}_{B}$. Once the optimal values of the Lagrange multipliers are determined, and provided that self-consistency is not achieved, we proceed to the next self-consistent iteration using the density matrix from the previous iteration. The process stops once the criteria for changes in the density matrix and total energy are met. Several consistency checks were performed to verify the robustness of our code. We have implemented this scheme in the Gaussian Development Version program $\underline{\underline{38}}$

Using this methodology, $E^{K S}(\theta)$ was calculated for small values of $\theta$ around $\theta=0$ and $\theta=180^{\circ}$ and magnetic exchange couplings were obtained from the quadratic coefficient of a polynomial fit. It is important at this point to mention that there are cases where existing approximate density functional methods have difficulties in representing the LS state $\left(\theta=180^{\circ}\right), \underline{1,11,15}$ The Kohn-Sham determinant in these cases correspond to a "brokensymmetry" solution that mixes two or more eigenfunctions of the $S^{2}$ operator. However, for the HS state $(\theta=0)$, it is customary accepted that approximate density functionals provide a reliable representation. Thus, even though for comparison purposes in the next Section we show our results using both the HS and LS states as reference, for the practical extraction of magnetic exchange couplings in the DFT framework, this method is expected to work more reliably using only the HS as the reference state.

\section{PROOF OF CONCEPT CALCULATIONS}

We first tested our methodology in the $\mathrm{H}-\mathrm{He}-\mathrm{H}$ linear model system with a distance $\mathrm{H}-\mathrm{He}$ of $1.625 \AA$, considering the outer $\mathrm{H}$ atoms as magnetic centers $A$ and $B$ $\left(S_{A}=S_{B}=1 / 2\right)$. In Table $\prod$ we show our results for the magnetic exchange couplings calculated from $d^{2} E^{K S} / d \theta^{2}$ using the $\theta=0\left(\left\langle S_{z}\right\rangle=1\right)$ state and the $\theta=180^{\circ}$ $\left(\left\langle S_{z}\right\rangle=0\right)$ state, $J^{H S}$ and $J^{L S}$, respectively. All calculations were carried out with the $6-311 \mathrm{G}^{* *}$ Gaussian basis set $\underline{39}$ For comparison, in Table $\prod$ we include results for the LSDA (Dirac exchange plus the parametrization of Wosko, Wilk, and Nusair ${ }^{40}$ for correlation), the BLYP realization of the GGA (Becke's 1988 functional ${ }^{41}$ for exchange and the correlation functional of Lee, Yang, and Parr ${ }^{42}$ ), and for the B3LYP $41,42,43,44$ hybrid functional. For all functionals, exchange couplings calculated from the energy derivatives, $J^{H S}$ and $J^{L S}$, are in very close agreement to the exchange coupling calculated from the energy difference, $J^{\Delta E}$. The difference can be attributed to both, the intrinsic accuracy of the numerical differentiation method and to the fact that $J^{H S}$ and $J^{L S}$ are expected to be identical to $J^{\Delta E}$ only in the case where
DFT describes the electronic system as an ideal Heisenberg model. The small discrepancy between $J^{H S}, J^{L S}$ and $J^{\Delta E}$ can be understood in terms of the localized nature of the magnetization on the $\mathrm{H}$ atoms in this model system and provides a measure of how well the electronic system mimics the behavior of an ideal Heisenberg model.

In Table \ we report $J^{\Delta E}$ as calculated from the SP formula, Eq. (2), since it offers a direct comparison between $J^{H S}$ and $J^{L S}$, and $J^{\Delta E}$. It is worth mentioning that in the ideal case of a perfect Heisenberg system, $\Delta E=E\left(\theta=180^{\circ}\right)-E(\theta=0)$ is related to $J^{H S}$ and $J^{L S}$ according to

$$
\Delta E=2\left(\frac{d^{2} E}{d \theta^{2}}\right)_{\theta=0}=-2\left(\frac{d^{2} E}{d \theta^{2}}\right)_{\theta=180^{\circ}} .
$$

Therefore, $d^{2} E / d \theta^{2}$ provides a measure of $\Delta E$ that can be evaluated without explicitly converging the selfconsistent procedure to the LS state.

TABLE I: Magnetic exchange couplings (in meV) calculated from energy derivatives and from energy differences for the $\mathrm{H}-\mathrm{He}-\mathrm{H}$ system.

\begin{tabular}{lrrr}
\hline \hline & LSDA & BLYP & B3LYP \\
\hline$J^{H S}=\frac{1}{2 S_{A} S_{B}}\left(\frac{d^{2} E}{d \theta^{2}}\right)_{\theta=0}$ & -95.8 & -74.0 & -60.8 \\
$J^{L S}=-\frac{1}{2 S_{A} S_{B}}\left(\frac{d^{2} E}{d \theta^{2}}\right)_{\theta=180^{\circ}}$ & -101.7 & -76.6 & -61.2 \\
$J^{\Delta E}=\frac{E\left(\theta=180^{\circ}\right)-E(\theta=0)}{4 S_{A} S_{B}}$ & -99.8 & -76.9 & -63.5 \\
\hline \hline
\end{tabular}

Our second proof of concept was carried out in the oxovanadium(IV) dimer $\left[\left(\mu-\mathrm{OCH}_{3}\right) \mathrm{VO}(\mathrm{ma})\right]_{2}$. This complex shows a strong antiferromagnetic coupling of about $-13.3 \mathrm{meV}$, as measured by temperature-dependent magnetic susceptibility experiments $\underline{\underline{45}}$ Here we employed Ahlrich's triple-zeta valence basis set for for the V atoms and Ahlrich's double-zeta valence basis for firstrow atoms $\frac{46,47}{4}$, as obtained from Ref. 48. This basis was shown to provide reliable results in practical cal-

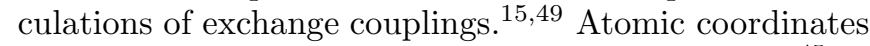
were taken from experimental crystallographic data $\stackrel{45}{\underline{I n}}$ Figs. 2 and 3 we present a plot of $E^{K S}$ as a function of $\theta\left(0 \leq \theta \leq 180^{\circ}\right)$ for LSDA and B3LYP, respectively. In both figures, $E^{K S}(\theta)$ follows closely a cosine function connecting the HS and LS extrema, indicating that both functionals capture the Heisenberg behavior of the oxovanadium complex. Related investigations in periodic systems using the LSDA can be found in the literature $.50,51$

For the plots shown in Fig. 2 and Fig. 3 we have chosen as magnetic centers $A$ and $B\left(S_{A}=S_{B}=1 / 2\right)$ both sets of $\mathrm{V}$ and apical $\mathrm{O}$ atoms since most of the spin density in this complex is localized on this moiety, as shown in Fig. 4. However, it is worth to mention that by choosing the metal atoms only as magnetic centers $A$ and $B$ the changes in the plots are unappreciable and the calculated 


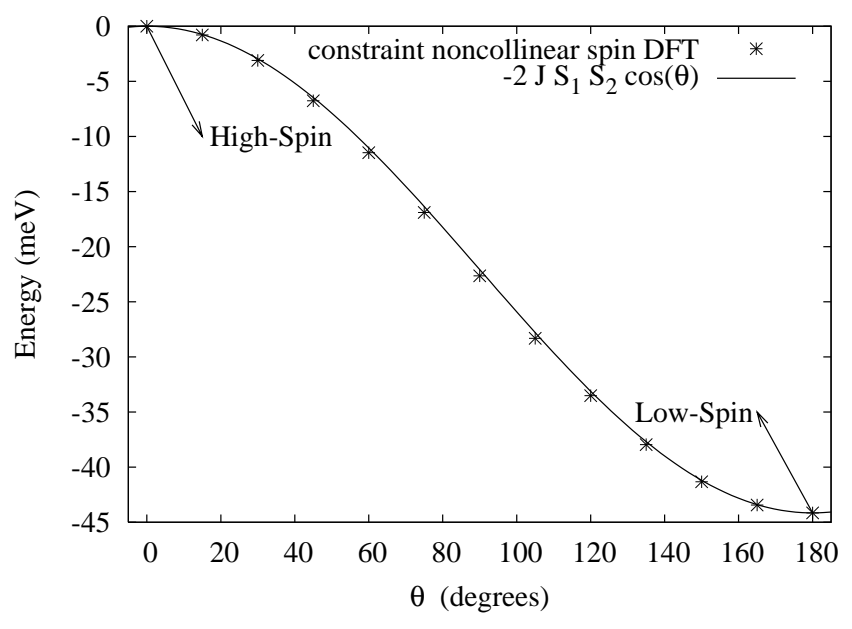

FIG. 2: LSDA energy change as a function of the angle between the local magnetic moments obtained from a constraint noncollinear spin density functional calculation in the oxovanadium complex (Fig. 4). The solid line shows the (ideal) cosine function connecting the AF and FM extrema.

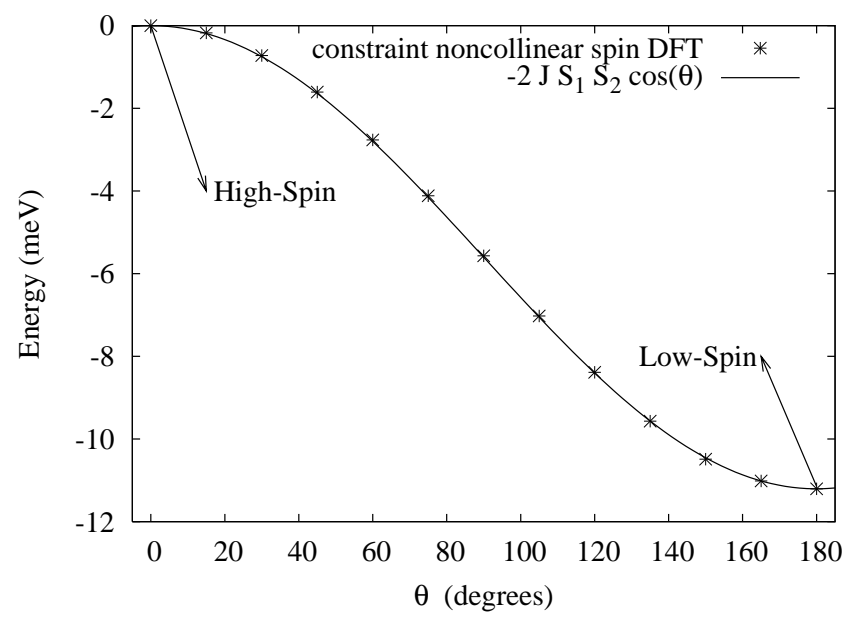

FIG. 3: Same as Fig. 2 for B3LYP.

magnetic exchange couplings $J^{H S}$ and $J^{L S}$ vary very little. For instance, for LSDA, magnetic exchange couplings change (in $\mathrm{meV}$ ) from $J^{H S}=-46.6$ to $J^{H S}=-46.9$ and from $J^{L S}=-41.9$ to $J^{L S}=-42.2$ when using the $\mathrm{V}$ and $\mathrm{O}$ atoms or the $\mathrm{V}$ atoms only, respectively. This indicates that our method is not sensitive upon a particular choice of the magnetic centers and shows, in this sense, robustness. One physical explanation for this fact is that the spin polarization of the light atoms surrounding a metal atom is "dragged" by the strong magnetic coupling with the neighbor metal center and therefore, it tends to align parallel (or antiparallel) to the magnetization of the metal center. For the case in study, if the constraint is applied on the $\mathrm{V}$ atoms only, the angle of the spin polarization on the apical $\mathrm{O}$ atom deviates from the direction of the constrain vectors by a maximum of
TABLE II: Magnetic exchange couplings (in meV) calculated from energy derivatives and from energy differences for the vanadium bimetallic complex. The corresponding experimental value is $-13.3 \mathrm{meV} .^{a}$

\begin{tabular}{lcr}
\hline \hline & LSDA & B3LYP \\
\hline$J^{H S}=\frac{1}{2 S_{A} S_{B}}\left(\frac{d^{2} E}{d \theta^{2}}\right)_{\theta=0}$ & -46.6 & -11.4 \\
$J^{L S}=-\frac{1}{2 S_{A} S_{B}}\left(\frac{d^{2} E}{d \theta^{2}}\right)_{\theta=180^{\circ}}$ & -41.9 & -11.2 \\
$J^{\Delta E}=\frac{E\left(\theta=180^{\circ}\right)-E(\theta=0)}{4 S_{A} S_{B}}$ & -44.1 & -11.3 \\
\hline \hline
\end{tabular}

${ }^{a}$ Taken from Ref. 45

only $2.75^{\circ}$ (for $\left.\theta=90^{\circ}\right)$.

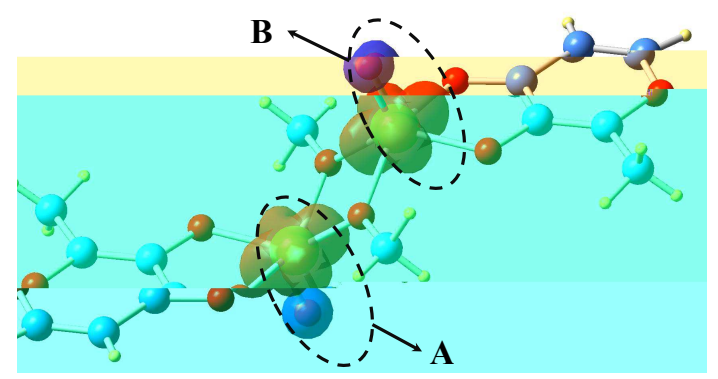

(a)

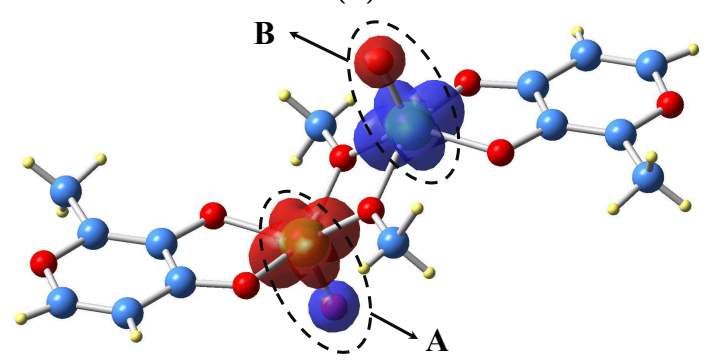

(b)

FIG. 4: Spin density isosurface of the HS (a) and LS (b) states of the oxovanadium complex (Fig. (4). Red corresponds to $\uparrow$ and blue to $\downarrow$. The isosurface represents a spin density of $0.01\left(\mathrm{Bohr}^{-3}\right)$. Magnetic centers $A$ and $B$ chosen for the calculations are also indicated.

A careful comparison of Fig. 2 and Fig. 3 evidences a larger deviation from the ideal cosine function for LSDA than for B3LYP. The largest differences from the cosine function are approximately $0.6 \mathrm{meV}$ and $0.04 \mathrm{meV}$ for LSDA and B3LYP, respectively, and occurs for $\theta=90^{\circ}$ in both cases. This is not surprising since LSDA yields electron (total and spin) densities more delocalized than B3LYP (the Löwdin atomic magnetic moments at the V atoms for the LS state are $1.00 \mu_{B}$ and $1.10 \mu_{B}$ for LSDA and B3LYP, respectively) and therefore, one can expect that the B3LYP energy follows the Heisenberg behavior more closely than its LSDA counterpart. Localization of the spin-density also reduces the calculated magnetic exchange couplings, as shown by Martin $\frac{52}{2}$, Ruiz $\underline{37,49}$, and demonstrated by Rudra et at. by explicitly constraining 
the local magnetization of the LS state 15 As shown in Table [II] The difference between $J^{H S}$ and $J^{L S}$ is $4.7 \mathrm{meV}$ for LSDA, while it is only $0.2 \mathrm{meV}$ for B3LYP. Thus, in contrast to the perfectly localized case, a more delocalized magnetization yields to larger deviations from the ideal Heisenberg behavior and hence greater differences between $J^{H S}$ and $J^{L S}$ and at the same time to larger exchange couplings.

\section{CONCLUSIONS}

We have proposed a method for the calculation of magnetic exchange couplings from noncollinear spin density functional calculations that employs the second derivative of the electronic energy of a single state with respect to a parameter, Eqs. (6) and (7). Within this approach there is no need to search for different self-consistent solutions of spin-states as it is commonly done in methods based on energy differences, such as the SP or NP methods, Eqs. (2) and (3). Our method utilizes perturbation theory for the evaluation of magnetic exchange couplings and therefore, in combination with standard analytic second derivatives techniques, it can potentially be used to compute exchange couplings very efficiently, opening the possibility of "black-boxifying" the extraction of magnetic exchange couplings from density functional theory calculations.

Our proof of concept calculations show very promising results. For the cases studied we found that our method reproduces exchange couplings obtained from the spin-projected approach based on energy differences. As expected from physical grounds, the agreement between both methods improves when the DFT description of the interaction between the magnetic centers is more Heisenberg-like. In this sense, the curve $E^{K S}(\theta)$ provides a quantitative measure of how well the electronic system mimics the behavior of an ideal Heisenberg model.

\section{ACKNOWLEDGMENTS}

This research was supported in part by an award from Research Corporation. J.E.P. acknowledges support from the President's Research Investment Fund (PRIF) and a start-up grant from Central Michigan University.
1 L. Noodleman, J. Chem. Phys. 74, 5737 (1981).

2 E. Ruiz, P. Alemany, S. Alvarez, and J. Cano, J. Am. Chem. Soc. 119, 1297 (1997).

3 J. Kortus, M. R. Pederson, T. Baruah, N. Bernstein, and C. S. Hellberg, Polyhedron 22, 1871 (2003).

${ }^{4}$ G. Christou, D. Gatteschi, D. N. Hendrickson, and R. Sessoli, MRS Bull. 25, 66 (2000).

5 P. Hohenberg and W. Kohn, Phys. Rev. B 136, 864 (1964).

${ }^{6}$ U. von Barth and L. Hedin, J. Phys. C: Solid State Phys. 5, 1629 (1972).

7 J. Kortus, C. S. Hellberg, and M. R. Pederson, Phys. Rev. Lett. 86, 3400 (2001).

8 J. Ribas-Arino, T. Baruah, and M. R. Pederson, J. Chem. Phys. 123, 044303 (2005).

${ }^{9}$ L. Noodleman and D. A. Case, Adv. Inorg. Chem. 38, 423 (1992).

10 L. Noodleman and E. R. Davidson, 109, 131 (1986).

11 E. Ruiz, J. Cano, S. Alvarez, and P. Alemany, 20, 1391 (1999).

12 L. Noodleman, J. G. Norman, J. H. Osborne, A. Aizman, and D. A. Case, Journal of the American Chemical Society 107, 3418 (1985).

13 E. Ruiz, A. Rodriguez-Fortea, J. Cano, S. Alvarez, and P. Alemany, 24, 982 (2003).

14 M. Nishino, S. Yamanaka, Y. Yoshioka, and K. Yamaguchi, J. Phys. Chem. A 101, 705 (1997).

15 I. Rudra, Q. Wu, and T. Van Voorhis, J. Chem. Phys. 124, 024103 (2006).

16 I. Rudra, Q. Wu, and T. Van Voorhis, Inorg. Chem. 46, 10539 (2007).

17 D. Dai and M.-H. Whangbo, J. Chem. Phys. 114, 2887 (2001).

18 A. E. Clark and E. R. Davidson, J. Chem. Phys. 115, 7382
(2001).

19 E. R. Davidson and A. E. Clark, J. Phys. Chem. A 106, 7456 (2002).

${ }^{20}$ W. Kohn and L. J. Sham, Phys. Rev. A 140, 1133 (1965).

21 A. I. Liechtenstein, M. I. Katsnelson, V. P. Antropov, and V. A. Gubanov, J. Magn. Magn. Mater. 67, 65 (1987).

22 J. Kübler, K.-H. Höck, J. Sticht, and A. R. Williams, J. Phys. F: Met. Phys. 18, 469 (1988).

${ }^{23}$ L. Nordsröm and D. J. Singh, Phys. Rev. Lett. 76, 4420 (1996).

24 T. Oda, A. Pasquarello, and R. Car, Phys. Rev. Lett. 80, 3622 (1998).

25 P. Kurtz, F. Förster, L. Nordsröm, G. Bihlmayer, and S. Blügel, Phys. Rev. B 69, 024415 (2004).

26 S. Yamanaka, D. Yamaki, Y. Shigeta, H. Nagao, Y. Yoshioka, N. Suzuki, and K. Yamaguchi, Int. J. Quantum Chem. 80, 664 (2000).

27 P. H. Dederichs, S. Blügel, R. Zeller, and H. Akai, Phys. Rev. Lett. 53, 2512 (1984).

28 J. Sticht, K.-H. Höck, and J. Kübler, J. Phys.: Condens. Matter 1, 8155 (1989).

29 L. M. Sandratskii, Adv. Phys. 1, 47 (1998).

30 J. E. Peralta, G. E. Scuseria, and M. J. Frisch, Phys. Rev. B 75, 125119 (2007).

31 N. R. M. Mayer, S. Kruger, J. Chem. Phys. 115, 4411 (2001).

32 J. E. Peralta and G. E. Scuseria, J. Chem. Phys. 120, 5875 (2004).

33 F. Wang and T. Ziegler, J. Chem. Phys. 121, 12191 (2004).

34 I. Malkin, O. L. Malkina, V. G. Malkin, and M. Kaupp, J. Chem. Phys. 123, 244103 (2005).

35 M. K. Armbruster, F. Weigend, C. van Wüllen, and W. Klopper, Phys. Chem. Chem. Phys. 10, 1748 (2008). 
36 P.-O. Löwdin, J. Chem. Phys. 18, 365 (1950).

37 E. Ruiz, J. Cirera, and S. Alvarez, Coord. Chem. Rev. 249, 2649 (2005).

38 Gaussian Development Version, Revision F.02, M. J. Frisch, G. W. Trucks, H. B. Schlegel, G. E. Scuseria, M. A. Robb, J. R. Cheeseman, J. A. Montgomery, Jr., T. Vreven, G. Scalmani, B. Mennucci, V. Barone, G. A. Petersson, M. Caricato, H. Nakatsuji, M. Hada, M. Ehara, K. Toyota, R. Fukuda, J. Hasegawa, M. Ishida, T. Nakajima, Y. Honda, O. Kitao, H. Nakai, X. Li, H. P. Hratchian, J. E. Peralta, A. F. Izmaylov, K. N. Kudin, J. J. Heyd, E. Brothers, V. Staroverov, G. Zheng, R. Kobayashi, J. Normand, J. L. Sonnenberg, S. S. Iyengar, J. Tomasi, M. Cossi, N. Rega, J. C. Burant, J. M. Millam, M. Klene, J. E. Knox, J. B. Cross, V. Bakken, C. Adamo, J. Jaramillo, R. Gomperts, R. E. Stratmann, O. Yazyev, A. J. Austin, R. Cammi, C. Pomelli, J. W. Ochterski, P. Y. Ayala, K. Morokuma, G. A. Voth, P. Salvador, J. J. Dannenberg, V. G. Zakrzewski, S. Dapprich, A. D. Daniels, M. C. Strain, O. Farkas, D. K. Malick, A. D. Rabuck, K. Raghavachari, J. B. Foresman, J. V. Ortiz, Q. Cui, A. G. Baboul, S. Clifford, J. Cioslowski, B. B. Stefanov, G. Liu, A. Liashenko, P. Piskorz, I. Komaromi, R. L. Martin, D. J. Fox, T. Keith, M. A. AlLaham, C. Y. Peng, A. Nanayakkara, M. Challacombe, W. Chen, M. W. Wong, and J. A. Pople, Gaussian, Inc., Wallingford CT, 2006.

39 R. Krishnan, J. S. Binkley, R. Seeger, and J. A. Pople, J.
Chem. Phys. 72, 650 (1980)

40 S. H. Vosko, L. Wilk, and M. Nusair, Can. J. Phys 58, 1200 (1980).

41 A. Becke, Phys. Rev. A 38, 3098 (1988).

42 C. Lee, W. Yang, and R. G. Parr, Phys. Rev. B 37, 785 (1988).

43 A. D. Becke, J. Chem. Phys. 98, 5648 (1993).

44 P. J. Stephens, F. J. Devlin, C. F. Chabalowski, and M. J. Frisch, J. Chem. Phys. 98, 11623 (1994), see also R. H. Hertwig and W. Koch, Chem. Phys. Lett. 268345 (1997).

45 Y. Sun, M. Melchior, D. A. Summers, R. C. Thompson, S. J. Rettig, and C. Orvig, Inorg. Chem. 37, 3119 (1998).

46 A. Schafer, Horn, and R. Ahlrichs, J. Chem. Phys. 97, 2571 (1992).

47 A. Schafer, C. Huber, and R. Ahlrichs, J. Chem. Phys. 100, 5829 (1994).

48 D. J. Feller, J. Comp. Chem. 17, 1571 (1996).

49 E. Ruiz, A. Rodriguez-Fortea, J. Tercero, T. Cauchy, and C. Massobrio, J. Chem. Phys. 123, 074102 (2005).

50 P. Kurz, G. Bihlmayer, K. Hirai, and S. Blügel, Phys. Rev. Lett. 86, 1106 (2001).

51 P. Novak, I. Chaplygin, G. Seifert, S. Gemming, and R. Laskowski, Comput. Mater. Sci. (2008), doi:10.1016/j.commatsci.2008.01.028.

52 R. L. Martin and F. Illas, Phys. Rev. Lett. 79, 1539 (1997). 